\title{
Therapeutic management of Bipolar Disorders Pierre Thomas
}

\author{
Address: Functional Neurosciences and Pathologies, Department of Psychiatry, University Lille 2, Hospital M. Fontan, Lille, France \\ from International Society on Brain and Behaviour: 3rd International Congress on Brain and Behaviour \\ Thessaloniki, Greece. 28 November - 2 December 2007 \\ Published: 17 April 2008 \\ Annals of General Psychiatry 2008, 7(Suppl I):S70 doi:I0.I I86/I744-859X-7-SI-S70
}

This abstract is available from: http://www.annals-general-psychiatry.com/content/7/SI/S70

(c) 2008 Thomas; licensee BioMed Central Ltd.

The therapeutic goal in bipolar disorder is to restore and maintain mental health with few relapses. To date, the efficacy of many mood stabilizer drugs has been assessed in patients with bipolar disorder at different stage of their disease. Mood stabilizers are defined as any medication that stabilizes acute manic, mixed, and depressive symptoms and does not induce alternate mood symptoms, and prevents against future relapses into manic, mixed, or depressive symptoms or episodes. Mood stabilizers generally include lithium, antiepileptics, and atypical antipsychotics. However medication tolerability and adherence issues are often reasons why patients do not achieve favorable outcomes. As such, clinicians need to systematically assess and address all the barriers to therapy adherence, a major risk factor for bipolar disorder management failure. As with all drugs, many treatments are associated with undesirable side effects. Patients with bipolar disorders share with those with schizophrenia some adverse effects including an increased risk for excessive weight gain, diabetes, and dyslipidemia (metabolic syndrome). Monitoring of drug levels, lipids, blood glucose, metabolic parameters, and weight is essential toward achieving the desired efficacy and preserving quality of life. Patients need to be educated as to the frequency of these side effects. Moreover, the patient's perceptions about the relative risks and benefits of treatment are a strong determinant of medication regimen adherence. The management of bipolar disorder requires a carefully planned strategic partnership between the patient, support systems, and all health care providers. 